\title{
The development of emergency medicine in Ethiopia
}

\author{
Fikre Germa, MD, FCFP (EM)
}

Ethiopia is an ancient country bordered by Kenya, Somalia, Sudan, and Eritrea. The mainly rural and predominantly young population of 80 million is expected to surpass 100 million by 2020. These demographics, along with decades of political turmoil and environmental degradation caused by overfarming, have resulted in widespread poverty ${ }^{1}$ and a dual burden of disease, both infectious and chronic, and too little health care infrastructure to respond appropriately. In addition to malaria, human immunodeficiency virus (HIV), and tuberculosis, the population is affected by cardiovascular diseases, type 2 diabetes, and asthma. Trauma is another major cause of morbidity and mortality, particularly trauma from motor vehicle accidents. Along with other health care improvements, Ethiopia is clearly in need of an emergency medicine (EM) system.

\section{CHALLENGES}

Ten years ago, there was essentially no infrastructure for EM in Ethiopia. Although this is beginning to change, many challenges remain. As in most developing countries, trained medical personnel and equipment are in short supply. In addition, Ethiopia's diverse terrain and spread-out settlements make delivering emergency care difficult. Lack of clear policies and continuing support for EM, high staff turnover, and woefully inadequate acute care space are other factors complicating care delivery. Ethiopia has a complex way of funding health care through a variety of national and international agencies. Although many countries and organizations generously donate medical equipment and supplies, a lack of hospital resources, bioengineering support, and in-service training means that these donations are not always used effectively.

\section{RECENT EM INITIATIVES}

Advocacy for EM began with the work of Dr. Tesfaye Mekonnen Bayleygne, an anesthesiologist and visionary leader at Black Lion Hospital in Addis Ababa. ${ }^{2}$ In 1999, an inaugural symposium on EM allowed the Addis Ababa Health Bureau, the Israeli medical community, the World Health Organization, and Addis Ababa University Faculty of Medicine to explore opportunities for formal training and discuss international partnerships to develop an EM system. Since then, various countries, including Canada, have become more involved in EM continuing medical education (CME) in Ethiopia. In one initiative, the Ethiopian North American Health Professionals Association (ENAHPA) and volunteer academics from Canadian and American universities hosted sessions in collaboration with the Ethiopian Medical Association. To date, over 600 Ethiopian health professionals have attended EM symposia focused on leadership, prehospital care, basic and advanced life support, and the evaluation and treatment of minor and major trauma.

In other initiatives, Canadian nurses have travelled to the country and taught triage and basic medical skills, an EM residency program has been set up at Black Lion Hospital, and a first-responder program has been established through the Fire and Emergency

From the Emergency Department, Brantford General Hospital, Brantford, ON, and the Department of Family Medicine, McMaster University, Hamilton, ON.

Correspondence to: Dr. Fikre Germa, Emergency Department, Brantford General Hospital, 200 Terrace Hill Street, Brantford, ON N3R 1G9; fikregerma130@ hotmail.com.

Submitted August 30, 2010; Revised December 9, 2010; Accepted December 15, 2010.

This article has been peer reviewed.

(c) Canadian Association of Emergency Physicians

CJEM 2011;13(6):411-412

DOI $10.2310 / 8000.2011 .110448$

Keywords: advocacy, continuing medical education, developing country, emergency medicine, Ethiopia 
Services in Addis Ababa. Teaching materials and medical equipment have also been donated by individual Canadian physicians, members of the Ethiopian medical diaspora, and a number of institutions, including Brantford General Hospital and Royal Alexandra Hospital in Edmonton, Alberta. Significant donations have included ultrasound machines, pulse oximeters, defibrillators, and core EM curricula.

\section{LIMITATIONS OF RECENT INITIATIVES}

The chief limitation with present EM development in Ethiopia has been the attempt to introduce a model developed in another country and culture. A specific system that works in another country will probably not work given Ethiopian culture and geography and the resources available. Stakeholders have not yet described the system they want to develop or formulated an overall strategy with articulated steps for achieving sustainable EM in Ethiopia. Before an effective EM system can be developed, all stakeholders must discuss Ethiopia's needs and agree on definitions and goals.

A closely related limitation is the lack of infrastructure and equipment for the initiatives now under way. This has resulted in a vastly overcrowded emergency department at Black Lion Hospital and ambulances without basic equipment being used by the Addis Ababa emergency services. It has also meant that supplies donated recently to one hospital could not help patients because staff did not know what they were or how to use them.

Another limitation involves the training of EM professionals by international $\mathrm{CME}$ providers. Although clinical and academic courses and intermittent EM training are offered in Ethiopia today, professional development has not been integrated into the country's medical education system. Without the development of current faculty, gaps will inevitably persist in the education of EM residents.

\section{RECOMMENDATIONS}

Our world is a highly interconnected one, and there are compelling economic and biosecurity reasons for building EM capacity in Ethiopia. ${ }^{3}$ Some progress has been made with the initiatives now under way, but more systems planning is needed to build a firm foundation for EM in Ethiopia. Although North American medical professionals can offer valuable expertise on systems design and future planning, the new African Federation for Emergency Medicine $(\mathrm{AFEM})^{4}$ would probably be a better source of development expertise, along with countries such as South Africa and Botswana. In addition, the International Federation for Emergency Medicine $(\text { IFEM) })^{5}$ would be a good source of some basic definitions that might be used when determining the scope of Ethiopia's EM system and the role to be played by various health professionals-from firstresponders to midlevel care providers and physicians.

To build capacity for delivering emergency care, Ethiopia cannot rely solely on expertise from outside Africa and on foreign faculty. Instead, local physicians need to be trained abroad and then encouraged to play a part in institutionalizing professional development in Ethiopia's hospitals and universities. Integrating CME in this way would help more Ethiopian health care workers acquire and maintain skills. These practitioners could then participate in medical education programs that train other health professionals-all with the goal of supporting an EM system that reflects Ethiopia's needs, culture, geography, and resources.

Competing interests: None declared.

\section{REFERENCES}

1. Oxford Poverty and Human Development Index. Country briefing: Ethiopia. Available at: www.ophi.org.uk/policy/ multidimensional-poverty-index/mpi-country-briefings/ (accessed September 7, 2010).

2. Bayleygne T, Shahar A, Tsadic AW, et al. An international training program to assist with establishing emergency medicine in Ethiopia. Ann Emerg Med 2000;36:378-82, doi:10.1067/mem.2000.110012.

3. Singer PA. Motivating action: why should Canadian physicians participate in research, education, or patient care in a developing country? Can Fam Physician 2007;53:1849-51.

4. African Federation for Emergency Medicine. Services. Available at: www.afem.info (accessed September 7, 2010).

5. International Federation for Emergency Medicine. About IFEM. Available at: www.ifem.cc/About_IFEM.aspx (accessed September 7, 2010). 